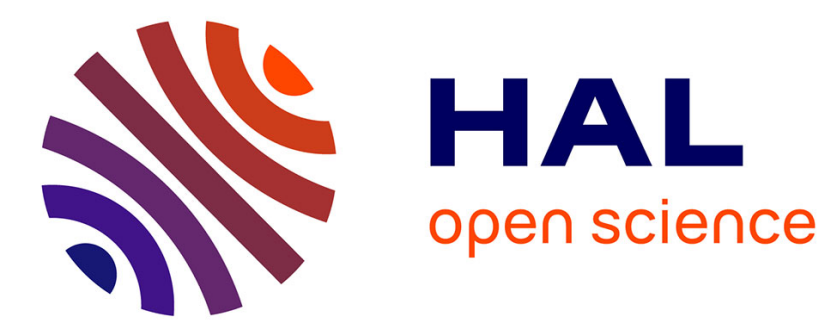

\title{
Écrire l'information: La translittéracie, un levier pour (ré-)concilier formes sociales et formes scolaires
}

\author{
Anne Cordier
}

\section{To cite this version:}

Anne Cordier. Écrire l'information: La translittéracie, un levier pour (ré-)concilier formes sociales et formes scolaires. Le Français Aujourd'hui, 2017, Ecriture numérique: Des usages sociaux aux formations, 196. halshs-01580459

\section{HAL Id: halshs-01580459 \\ https://shs.hal.science/halshs-01580459}

Submitted on 1 Sep 2017

HAL is a multi-disciplinary open access archive for the deposit and dissemination of scientific research documents, whether they are published or not. The documents may come from teaching and research institutions in France or abroad, or from public or private research centers.
L'archive ouverte pluridisciplinaire HAL, est destinée au dépôt et à la diffusion de documents scientifiques de niveau recherche, publiés ou non, émanant des établissements d'enseignement et de recherche français ou étrangers, des laboratoires publics ou privés. 
Cordier, Anne. Écrire l'information: La translittéracie, un levier pour (ré-)concilier formes sociales et formes scolaires. Le Français Aujourd'hui, mars 2017, n¹96, p.3545.

\title{
Écrire l’information : La translittéracie, un levier pour (ré-)concilier formes sociales et formes scolaires
}

\author{
Anne CORDIER, ESPÉ-Université de Rouen Normandie, UMR 6590 ESO
}

\section{Introduction}

Si écrire la recherche d'information est fondamentalement une pratique académique, laquelle s'illustre dans l'imaginaire collectif par le traditionnel exposé, parfois accompagné de le non moins traditionnelle "affiche", l'introduction des technologies de l'information et de la communication dans le processus d'écriture favorise de nouvelles formes d'écriture ou de ré-écriture, bousculant les traditions intellectuelles. Chercheuse en Sciences de l'Information et de la communication, impliquée successivement dans deux groupes de recherche ayant travaillé la notion de translittéracie ${ }^{1}$, nous avons pu mesurer, au sein des terrains investigués, la portée à la fois sociale et éducative de cette notion. La translittéracie n'est pas seulement la capacité à "lire, écrire et interagir à travers une variété de plateformes " (Thomas et al 2007), elle implique aussi - nous allons le voir plus loin - une vision socioconstructiviste de l'apprentissage et permet de considérer pleinement

\footnotetext{
${ }^{1}$ Projet AIR 2012 nommé «TRANS-I (pour « Translittéracie Informationnelle : Vers une nouvelle grammaire de l'information »), dirigé par Vincent Liquète, financé par l'Institut des Sciences de la Communication du CNRS. Présentation du projet : http://www.iscc.cnrs.fr/spip.php?article1706

Projet ANR TRANSLIT («La translittéracie comme horizon de convergence des littératies médiatiques, infodocumentaires et informatiques ») : http://www.agence-nationale-recherche.fr/?Projet=ANR-12-CULT-0004

${ }^{2}$ Voici la definition complète de la translittéracie proposée par Sue Thomas et ses collègues en 2007 : «Transliteracy is the ability to read, write and interact across a range of platforms, tools and media from signing and orality through handwriting, print, TV, radio and film, to digital social networks". Thomas, Sue et al. "Transliteracy: Crossing divides ». [En ligne]. 3 Décembre 2007, Vol. 12, $\mathrm{n}^{\circ}$ 12, Disponible sur : http://www.uic.edu/htbin/cgiwrap/bin/ojs/index.php/fm/article/view/2060/1908
} 
Cordier, Anne. Écrire l'information: La translittéracie, un levier pour (ré-)concilier formes sociales et formes scolaires. Le Français Aujourd'hui, mars 2017, n¹96, p.3545.

l'élève non comme un apprenant mais comme un être social aux prises avec des réalités quotidiennes et académiques complexes.

Dans cette contribution, nous souhaitons documenter le rapport à l'écriture de la recherche d'information que l'école développe, en lien avec les formes sociales d'écriture qui sont quotidiennement celles éprouvées par les adolescents. Comment se redessinent les contours du processus d'écriture de l'information aujourd'hui ?

Dans un premier temps, nous présenterons les contextes épistémologique et méthodologique de notre réflexion. Dans un second temps, nous montrerons combien le processus d'écriture de la recherche d'information apparaît aujourd'hui ré-agencé et enrichi sous l'influence double de formes sociales et de dispositifs institutionnels. Enfin, nous analyserons les nouvelles compétences scripturales qu'il nous semble important de développer à l'heure de la translittéracie lorsqu'il s'agit de penser ensemble écriture et activité informationnelle.

\section{Contextes épistémologique et méthodologique}

\section{Ce que la notion de translittéracie apporte à la dialectique SIC/Écriture}

Si la notion de translittéracie, définie par l'équipe de la chercheuse britannique Sue Thomas en 2007 comme une capacité à exploiter à des fins de lecture, d'écriture et de communication, une variété d'outils et de supports (Thomas et al. 2007), a attiré l'attention des chercheurs français en Sciences de l'Information et de la Communication (SIC), c'est avant tout parce qu'elle a pointé la convergence médiatique à l'œuvre dans notre société. 
Cordier, Anne. Écrire l'information: La translittéracie, un levier pour (ré-)concilier formes sociales et formes scolaires. Le Français Aujourd'hui, mars 2017, n¹96, p.3545.

Une convergence qui est aussi, dans le cadre du système éducatif français, une convergence des littéracies, permettant de penser les liens et interrelations entre trois domaines principaux relevant de la considération des types d'information: la culture de l'information, la culture médiatique, et l'informatique (Serres 2012). Les chercheurs en SIC mais aussi les professionnels de l'Information-Documentation (professeurs documentalistes dans l'enseignement secondaire, particulièrement, mais aussi bibliothécaires) y ont vu l'opportunité de repenser les formations des élèves et des usagers pour une appréhension davantage sociale de l'activité informationnelle. Ainsi la définition de la translittéracie a connu un enrichissement très important en France, notamment à travers les projets Trans-I et TransLit, qui ont travaillé cette notion à la fois sur le plan épistémologique et sur le plan méthodologique et empirique.

Plus encore, à nos yeux, la translittéracie porte une vision résolument constructiviste de l'apprentissage, amenant à s'intéresser aux transferts entre domaines d'apprentissage formels (académiques) et non formels (non validés par l'institution scolaire, sphère domestique et familière), ainsi qu'aux ponts à la fois cognitifs et de pratiques entre les cadres d'usages des acteurs.

La translittéracie invite à analyser ce qui se joue précisément entre les individus dans certaines situations et/ou avec certains dispositifs, la manière dont se construisent des savoirs dans la relation sociale, par la négociation et la coconstruction d'activités. Elle est donc tout à la fois, comme le préfixe "trans » le laisse entendre, transversalité de compétences et connaissances communes aux contextes info-communicationnels, transformation des situations informationnelles par des pratiques et processus personnels, transition de la 
Cordier, Anne. Écrire l'information: La translittéracie, un levier pour (ré-)concilier formes sociales et formes scolaires. Le Français Aujourd'hui, mars 2017, n¹96, p.3545.

vision d'une connaissance individuelle à une connaissance partagée, collective, et transgression des normes/standards académiques (Liquète 2012).

\section{Méthodologie de recherche}

Notre perspective est celle d'une approche écologique des pratiques informationnelles dans l'organisation scolaire, attentive au rôle du contexte et à l'influence des positionnements des acteurs sociaux au sein de la situation observée. Concrètement, nous avons mené des investigations de terrain ${ }^{3}$ dans un lycée public français, situé dans l'Académie de Lille, au sein d'un réseau urbain économiquement peu favorisé. Nous avons suivi durant une année (18 semaines de séances de $2 \mathrm{~h}$ hebdomadaires et passages oraux) le dispositif institutionnel Travaux Personnels Encadrés (TPE) tel que mis en place auprès d'une classe de 36 élèves de Première Section Économique et Sociales (SES), ainsi qu'un projet déployé en classe de Seconde (31 élèves) au sein de l'option Littérature et Société ( $2 \mathrm{~h}$ par semaine pendant un trimestre), amenant les élèves à travailler autour du mythe de Babel à travers les arts. Puis l'année suivante, notre attention s'est portée sur un projet Anglais-InformationDocumentation (1h par demi-groupe, de septembre à fin avril), au sein duquel les élèves scolarisés en Terminale Littéraire (au nombre de 36 ) ont été engagés à réaliser, en groupe, un carnet de voyage numérique.

Pour chacune de ces investigations, nous avons adopté le même protocole. Dans un premier temps, nous nous sommes immergée pleinement dans les situations info-communicationnelles mises en place, et avons procédé à un recueil de données reposant sur une combinaison de techniques

\footnotetext{
${ }^{3}$ Nous tenons à préciser que les données dont il est fait état ici ont été recueillies par nous-même dans le cadre d'un protocole collectivement élaboré.
} 
Cordier, Anne. Écrire l'information: La translittéracie, un levier pour (ré-)concilier formes sociales et formes scolaires. Le Français Aujourd'hui, mars 2017, n¹96, p.3545.

qualitatives, à savoir observation distanciée des séances et des activités engagées par les groupes d'élèves pris pour étude, au moyen d'une grille définie, et captation photographique de "moments forts", de traces de l'organisation de l'activité. Pendant ces observations, équipée d'un dictaphone numérique enregistrant la totalité de la séance, nous avons mené ponctuellement des entretiens d'explicitations individuels ou collectifs avec les élèves, ou les enseignants, lorsqu'un élément retenait particulièrement notre attention. Faire appel à cette "prise de parole impliquée ou incarnée " (Vermersch 1996) permet de dégager des logiques d'action aux yeux des acteurs, en faisant référence directement à une situation en train d'être vécue et agencée. Dans un second temps, nous avons mené des entretiens semidirectifs, individuels ou collectifs, avec les acteurs observés (enseignants et lycéens).

\section{De nouvelles formes scolaires pour écrire l’information}

Morgan, 16 ans, élève en classe de Seconde est catégorique: «Clairement, les recherches d'info, souvent c'est écrire ce que t'as lu, mais en trouvant d'autres mots. Alors, tu fouilles dans le dico des synonymes pour dire de pas être étiqueté « copieur devant l’Éternel !». Le jeune lycéen dénonce ici la tradition académique de la recherche d'information, qui est faite de règles de ré-écriture formalisées (elle constitue une étape de l'activité de recherche documentaire, telle que formalisée dans les référentiels de compétences 
Cordier, Anne. Écrire l'information: La translittéracie, un levier pour (ré-)concilier formes sociales et formes scolaires. Le Français Aujourd'hui, mars 2017, n¹96, p.3545.

documentaires, qui modélisent et décomposent l'activité de recherche documentaire de manière extrêmement linéaire ${ }^{4}$ ).

\section{L'influence de formes sociales}

Rompre avec cette tradition n'est pas aisé car est liée à l'acte de réécriture l'évaluation de la compréhension de l'information soumise à reformulation. Les enseignants se trouvent sans cesse en posture de vigilance et de contrôle, afin de déterminer ce qui, dans le travail produit par l'élève, relève d'un "véritable travail " (Pr-Let) de réécriture et d'appropriation des contenus. Une forte crispation est observable autour des pratiques d'écriture de la recherche d'information, au premier rang desquels la pratique du copiécollé, qui traverse les discours agacés des enseignants.

Si cette pratique du copié-collé est sans conteste décuplée avec les outils informatiques, qui la rendent plus facile et plus rapide, pour autant la réduire à une simple manipulation technique nous semble erroné. Les travaux de Nicole Boubée sur l'activité de recherche d'information en ligne chez des collégiens et lycéens démontrent que le copié-collé fait partie intégrante de l'effectuation de l'activité informationnelle, la chercheuse insistant sur le rôle cognitif de cette activité (Boubée 2007).

La stigmatisation dont fait l'objet cette technique du copié-collé dans le monde social est mal comprise par les élèves eux-mêmes. Beaucoup éprouvent avant tout de réelles difficultés à percevoir "le mal » : "Être fidèle, ce n'est pas copier ", affirme Amélie, $1^{\text {ère }}$ ES. Au-delà, l'opposition entre un acte

\footnotetext{
${ }^{4}$ FADBEN (1997). Compétences en Information-Documentation : référentiel. Paris: FADBEN-CDI, 28p. (Médiadoc/Les dossiers).
} 
Cordier, Anne. Écrire l'information: La translittéracie, un levier pour (ré-)concilier formes sociales et formes scolaires. Le Français Aujourd'hui, mars 2017, n¹96, p.3545.

d'écriture-réécriture devenu monnaie courante sur les réseaux socionumériques par exemple et un acte de reformulation académiquement exigé crée une véritable ligne de tension entre les acteurs dans l'école. Nous avons pu noter une nette différenciation des usages de copié-collé selon les sphères de pratiques considérées. Prenons à titre d'exemple Thomas, élève en $1^{\text {ère }} E S$, qui nous explique devoir prendre garde à ne pas copier-coller lors de sa recherche d'information menée au cours de la séance TPE, et justifie ainsi : « Là, c'est pas pareil, c'est pour le lycée (...) C'est ce qu'on attend de nous ». On notera ce " on " dans "ce qu'on attend de nous ", qui est particulièrement intéressant ici. En effet, ce "on" fait moins référence aux enseignants encadrant le dispositif, notamment le professeur documentaliste qui a d'ailleurs des pratiques plutôt souples et ne tient pas de discours prescriptif fort sur la manière d'effectuer une recherche d'information, que à la présence d'une "instance-tiers », pour reprendre le concept de Patrick Charaudeau. Cette « instance-tiers » médiatise la conversation, selon la théorie pragmatique des actes de langage : ce Tiers, absent, symbolique, confirme que "parler, [c'est] toujours parler sous couvert de discours de référence implicites, d'imaginaires qui font office de norme sociale, au nom desquels les énoncés prennent leur valeur sémantique " (Charaudeau $2004: 30$ ). Thomas énonce ainsi dans cette situation observée une doxa qui lui paraît applicable à toutes situations scolaires, formulant une norme véhiculée, intériorisée, voire même un discours attendu.

Les lycéens apparaissent ainsi pris entre des logiques institutionnelles, qui se heurtent aux logiques individuelles et sociales de pratiques de l'information. Le risque d'une telle dichotomie est de provoquer de nettes 
Cordier, Anne. Écrire l'information: La translittéracie, un levier pour (ré-)concilier formes sociales et formes scolaires. Le Français Aujourd'hui, mars 2017, n¹96, p.3545.

différenciations entre les sphères formelles et non formelles... et donc de ne pas donner à l'enseignant la possibilité réellement d'agir avec efficacité sur ces pratiques, puisqu'elles sont rendues invisibles. C'est pourquoi il apparait pertinent d'exploiter le copié-collé comme ressource pour repenser les formes scolaires d'écriture de l'information, en lien avec les pratiques multimédiatiques des acteurs.

En dehors du monde scolaire se développent des pratiques très riches appuyées sur la technique du copié-collé, telles que celle du mash-up, genre hybride - musical à ses origines - qui consiste à assembler plusieurs extraits de créations pour donner naissance à une création originale. Mash-up et multimédia s'associent d'ailleurs pleinement depuis 2011 et l'instauration d'un Festival du Mashup Film qui met à l'honneur des créations associant plusieurs morceaux de musique ou fragments cinématographiques pour composer un objet artistique hybride ${ }^{5}$. Nous avons pu assister à des projets pédagogiques favorisant cette exploitation des formes sociales de restitution/production de l'information. Parmi ces projets, la réalisation de vidéos associant textes, images et sons, dans le cadre d'une séquence de l'option Littérature et Société intitulée " Racontez-nous votre Babel », ou encore la réalisation d'un carnet de voyage numérique exigeant des élèves de Terminale le croisement entre musiques, textes (en anglais) et photos prises par eux-mêmes. Des projets jugés très favorablement par les élèves, à l'instar de Zoé, scolarisée en Terminale : «On dit toujours que ce qu'on fait avec le numérique, c'est nul ou que ça sert à rien. Ben là, la preuve que si, si ça peut servir à quelque chose, même si c'est pas grand-chose! ». Réaliser cette production infocommunicationnelle scénarisée, appuyée sur des compétences développées

\footnotetext{
${ }^{5}$ Site du Mashup Film Festival : http://www.mashupfilmfestival.fr/presentation/
} 
Cordier, Anne. Écrire l'information: La translittéracie, un levier pour (ré-)concilier formes sociales et formes scolaires. Le Français Aujourd'hui, mars 2017, n¹96, p.3545.

hors l'école et des techniques de réécriture de l'information socialement éprouvées, a engendré aussi chez ces lycéens un fort sentiment de personnalisation du travail de recherche d'information : " J'ai adoré faire ça, il y avait de vrais morceaux de moi dans ce travail ! ", confie Morgan, 16 ans, qui souligne aussi le plaisir ressenti dans cette activité académique pourtant souvent anxiogène pour les jeunes.

\section{L'influence de dispositifs institutionnels}

Il ne faudrait cependant pas croire que les nouvelles formes scolaires émergeant pour écrire l'information ne sont pas aussi destinées à nourrir les pratiques sociales. S'impose en effet pour ces jeunes acteurs inscrits dans un milieu informationnel sans cesse enrichi la nécessité de discriminer au sein des ressources à leur disposition et de conscientiser leur activité informationnelle. Développer des compétences d'écriture réflexive de la démarche de recherche d'information est un enjeu majeur pour le professeur documentaliste du lycée où nous avons enquêté, qui s'appuie sur le carnet de bord dans le cadre des TPE : « C'est important d'avoir une réflexion sur ce qu'on fait, d'essayer de comprendre pourquoi on fait telle chose, comment on $s^{\prime} y$ prend, de ne pas fonctionner au petit bonheur la chance, c'est un outil de structuration de la pensée, vraiment, et de la démarche de recherche par conséquent ».

La mise en place du carnet de bord, comme outil de soutien à la démarche réflexive, nous semble renforcer la vision d'un « individu-plus » tel que pensé par Perkins (Perkins, 1995). Il est véritablement un document-action, au sens où l'entendent Sylvie Dalbin et Brigitte Guyot (Dalbin, Guyot, 2007), parce que c'est l'action qui est engagée ou visée dans la production ou l'exploitation du document. Il s'agit de penser son action propre dans l'organisation, à la fois au 
Cordier, Anne. Écrire l'information: La translittéracie, un levier pour (ré-)concilier formes sociales et formes scolaires. Le Français Aujourd'hui, mars 2017, n¹96, p.3545.

sens large et au sens plus restreint de la mini-organisation constituée par le groupe de travail.

Dans cette perspective, nous enjoignons à focaliser l'activité informationnelle sur le processus d'écriture réflexive, opérant ainsi un changement de paradigme : c'est en effet se départir d'une vision souvent trop restrictive dans le monde de l'enseignement de la recherche d'information, laquelle est souvent considérée comme un simple moyen de parvenir à une production sujette à évaluation. Il s'agirait alors de penser la réalisation de la production comme reflétant non pas le "résultat de la recherche", mais le processus informationnel en lui-même. En quelque sorte, il s'agirait de penser une écriture de son propre processus informationnel, à travers la construction d'un parcours réflexif documenté.

\section{Nouvelles compétences scripturales à l'ère de la Translittéracie}

On le voit, à l'ère de la translittéracie où les frontières entre modes d'écriture sociales et scolaires sont poreuses, notamment, de nouvelles compétences scripturales émergent. Et les acteurs, tous statuts confondus, sont unanimes, effectuant strictement le même constat : " C'est beaucoup plus difficile »(Pr-Doc, Pr-Let, Pr-Ang, Morgan, Amélie, Flavien).

\section{" Digital natives ", à vos souris !}

Si c'est " beaucoup plus difficile", pour les élèves en tout cas, c'est d'abord parce que parmi ces compétences scripturales, beaucoup sont implicitement considérées comme maîtrisées aux yeux des enseignants. En effet, ces derniers ne manquent pas lors des entretiens de souligner les 
Cordier, Anne. Écrire l'information: La translittéracie, un levier pour (ré-)concilier formes sociales et formes scolaires. Le Français Aujourd'hui, mars 2017, n¹96, p.3545.

compétences développées par leurs élèves en matière de technique numérique. Cela les conduit souvent à ne pas percevoir précisément leur rôle dans l'accompagnement de l'activité informationnelle qui est de fait fortement instrumentée de nos jours. Sont ainsi mis à jour des imaginaires des pratiques numériques adolescentes qui biaisent complètement l'appréhension par les enseignants des compétences et connaissances effectives de leur public (Cordier, 2015).

La croyance d'une dextérité homogène a la vie dure dans le corps enseignant, comme dans l'ensemble de la société d'ailleurs. Nous avons pourtant observé plusieurs jeunes, filles et garçons, en butte à de sérieuses déconvenues face aux exigences techniques que requiert l'activité informationnelle. Parmi ces jeunes, Armelle, 17 ans : "Le problème, c'est que les profs, ils considèrent qu'on doit connaître, et qu'on sait faire parce que nous, on est " les Jeunes du $21^{\text {ème }}$ siècle ", vous voyez, genre les branchés, quoi. Mais concrètement, moi, j'avais jamais vu ça avant, chez moi je fais pas ça, donc je sais pas l'utiliser. C'est pas parce que j'ai un I-Phone que je suis calée ».

Une autre croyance persiste, celle qui consiste à penser que les adolescents " maîtrisent " pleinement, alors que leurs pratiques sont majoritairement situées, dépendantes d'un contexte sociotechnique précis. Mégane se souvient avoir été "angoissée " - c'est son mot - au début du projet AnglaisDocumentation, lorsque les enseignants ont exigé un carnet de voyage numérique : "Je me demandais vraiment comment on allait s'en sortir, parce que à la maison des petits albums pour le plaisir, oui je sais faire, mais aller plus loin, et pour le lycée, c'est autre chose ". 
Cordier, Anne. Écrire l'information: La translittéracie, un levier pour (ré-)concilier formes sociales et formes scolaires. Le Français Aujourd'hui, mars 2017, n¹96, p.3545.

\section{Une complexification cognitive du processus d'écriture de l'information}

À l'ère de la translittéracie, on assiste à une complexification cognitive du processus d'écriture de l'information. Les compétences cognitives convoquées pour mener à bien les projets pédagogiques observés en classe sont nombreuses et particulièrement exigeantes pour les jeunes chercheursproducteurs d'information: décentration cognitive et métacognition, notamment, sont requises tout au long du processus.

La maîtrise de la langue sous-tendue par cette conception translittéracique de l'écriture informationnelle - du langage pour soi au langage partagé,

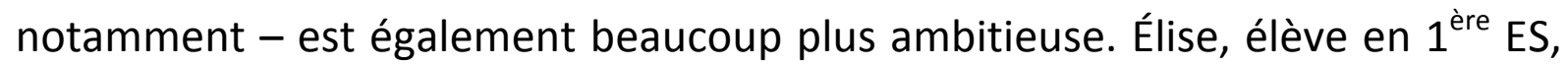
le confirme: "Le truc, c'est que d'habitude, tu cherches juste à remplacer un mot par un autre, pour coller le plus au texte de départ, et être certain de pas te tromper. Là, si tu n'as pas compris un truc, ça se voit tout de suite ! Il faut donc être plus vigilant, et c'est un travail qui est dur, parce qu'il faut avoir le vocabulaire qui va avec ". N'oublions pas que ce "langage " n'est de surcroît pas que scriptural, mais est aussi celui de l'image, fixe et animée, du son... l'ensemble devant être conjugué pour former un tout informationnel cohérent et maîtrisé.

Enfin, sortir de l'imitation, de ce mimétisme informationnel traditionnel, suppose aussi de faire véritablement preuve de créativité, enjeu social contemporain lié à la capacité à être dans le monde et à agir dans ce monde, individuellement ou collectivement (Sahlberg, 2011), mais aussi à sortir des sentiers battus, faire œuvre de création de soi en se situant dans le monde (Cordier, 2016). Les lycéens rencontrés durant ces trois projets pédagogiques apprécient d'être moins "passifs " dans le travail d'écriture de l'information, 
Cordier, Anne. Écrire l'information: La translittéracie, un levier pour (ré-)concilier formes sociales et formes scolaires. Le Français Aujourd'hui, mars 2017, n¹96, p.3545.

faisant la part belle à l'initiative et l'engagement, mais ils reconnaissent aussi que cela sollicite leur capacité à faire preuve d'imagination, mais aussi d'implication: "Tu dois être beaucoup plus concerné, c'est ce qu'on attend de toi » (Guillaume, 17 ans, Terminale).

\section{Conclusion : " Libérer le geste d'écriture »!}

Ainsi, nos recherches contribuent à mettre à jour des processus d'écriture en tensions, lycéens et enseignants (re)négociant des pratiques infocommunicationnelles en raison des influences translittéraciques. Ces constats nous conduisent ainsi à penser autrement les compétences d'écriture de la recherche d'information.

Placer les élèves en situation non plus de lecteurs-régurgiteurs d'information mais en situation de lecteurs-producteurs de l'information constitue un enjeu tant éducatif que social à l'heure où la compétence de publication relève non plus d'une compétence scolaire strictement encadrée mais d'une compétence de culture générale, incluant vies quotidienne et professionnelle. Conscientiser ses processus d'écriture et les transferts potentiels de compétence et de connaissances est une impérieuse nécessité aujourd'hui, alors que les écosystèmes informationnels des acteurs se complexifient et que les formats éditoriaux en ligne sont porteurs de logiques économiques souvent invisibles. II en va de l'émancipation de chacun-e au sein des dispositifs info-communicationnels. À ces seules conditions pouvons-nous espérer « libérer le geste d’écriture » (Jeanneret, Souchier, 2015). 
Cordier, Anne. Écrire l'information: La translittéracie, un levier pour (ré-)concilier formes sociales et formes scolaires. Le Français Aujourd'hui, mars 2017, n¹96, p.3545.

\section{Bibliographie}

BOUBÉE, N. (2007). Des pratiques documentaires ordinaires: analyse de l'activité de recherche d'information des élèves du secondaire. Thèse en SIC, Université Toulouse II.

CHARAUDEAU, P. (2004). Tiers, où es-tu ? à propos du tiers du discours. In P. Charaudeau, R. Montes, Rosa (dir.), La voix cachée du tiers : des non-dits dans le discours (pp. 20-41). Paris : L'Harmattan.

CORDIER, A. (2011). Formaliser l'activité de recherche d'information: les modèles et modélisations à l'épreuve des pratiques effectives. Colloque scientifique international du Réseau MUSSI. 15-17 juin, Toulouse.

CORDIER, A. (2011). Imaginaires, représentations, pratiques formelles et non formelles de la recherche d'information sur Internet: Le cas d'élèves de $6^{\text {ème }}$ et de professeurs documentalistes. Thèse en SIC, Université Lille 3. Disponible sur : http://tel.archives-ouvertes.fr/docs/00/73/76/37/PDF/THESE_Volume_1.pdf CORDIER, A. (2015). Grandir Connectés: Les adolescents et la recherche d'information. Caen : C \& F Éditions.

CORDIER, A. (2016). Et pourtant ils créent... : Quand la créativité des pratiques informationnelles juvéniles enrichit les pratiques pédagogiques, et réciproquement. Colloque EntreLacer: Littératie, Arts et Culture des Jeunes, ACFAS, 9-10 mai, Montréal (Canada).

DALBIN, S., GUYOT, B. (2007). Documents en action dans une organisation : des négociations à plusieurs niveaux ». Études de Communication, 30, 55-70. JEANNERET, Y., SOUCHIER, E. (2005). L'énonciation éditoriale dans les écrits d'écran. Communication \& Langages, 145, 03-15. 
Cordier, Anne. Écrire l'information: La translittéracie, un levier pour (ré-)concilier formes sociales et formes scolaires. Le Français Aujourd'hui, mars 2017, n¹96, p.3545.

LIQUÈTE, V. (2012). Can one speak of an "information transliteracy"? Colloque International "Media and Information Literacy for Knowledge Societies", Moscou (Russie).

PERKINS, D. N. (1995). L'individu-plus : une vision distribuée de la pensée et de l'apprentissage. Revue Française de Pédagogie, 111, 57-71.

SAHLBERG, P. (2011). Creativity and innovation through lifelong learning. Lifelong Learning in Europe, 16.

SERRES, A. (2012). Repères sur la translittéracie. Séminaire du GRCDI. Disponible $\quad$ sur $\quad: \quad$ http://culturedel.info/grcdi/wpcontent/uploads/2012/09/Seminaire-GRCDI-2012_texte-A.Serres.pdf THOMAS S. et al. (2007). Transliteracy: Crossing divides. First Monday, 12. Disponible sur

http://www.uic.edu/htbin/cgiwrap/bin/ojs/index.php/fm/article/view/2060/1 908

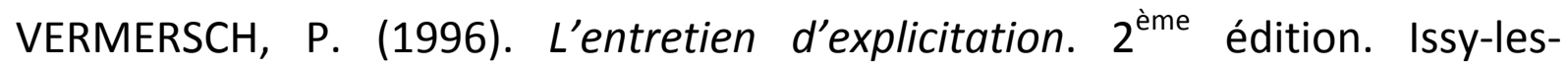
Moulineaux : ESF. 